\title{
Oral Squamous Cell Carcinoma and Associated Risk Factors in Jazan, Saudi Arabia: A Hospital Based Case Control Study
}

\author{
Mir Faeq Ali Quadri'*, Fahd Alharbi², Amal Mansoor S Bajonaid ${ }^{1}$, Ibtisam \\ Hussain Y Moafa ${ }^{1}$, Abubakker Al Sharwani ${ }^{3}$, Abdulwahab Hussain A Alamir ${ }^{1}$
}

\begin{abstract}
Background: Oral cancer is the third most common malignancy in Saudi Arabia, the highest incidence of which is reported from Jazan province. The objective of this study was to evaluate the association of various locally used substances, especially shamma, with oral cancer in the Jazan region of Saudi Arabia. Materials and Methods: A hospital-based case-control study was designed and patient records were scanned for histologically confirmed oral cancer cases. Forty eight patients who were recently diagnosed with oral cancer were selected as cases. Two healthy controls were selected for each observed case and they were matched with age $(+/-5$ years) gender and location. Use of different forms of tobacco such as cigarettes, pipe-smoking and shamma (smokelesstobacco) was assessed. Khat, a commonly used chewing substance in the community was also included. Descriptive analysis was first performed followed by multiple logistic regression (with and without interaction) to derive odds ratios (ORs) and 95\% confidence interval (CIs). Results: Mean age of the study sample (56\% males and $44 \%$ females) was 65.3 years. Multinomial regression analysis revealed that shamma use increased the odds of developing oral cancer by 29 times $(\mathrm{OR}=29.3 ; 10.3-83.1)$. Cigarette $(\mathrm{OR}=6.74 ; 2.18-20.8)$ was also seen to have an effect. With the interaction model the odds ratio increased significantly for shamma users $(O R=37.2 ; 12.3-113.2)$ and cigarette smokers $(\mathrm{OR}=10.5 ; 2.88-3.11)$. Khat was observed to have negative effect on the disease occurrence when used along with shamma $(O R=0.01 ; 0.00-0.65)$. Conclusions: We conclude that shamma, a moist form of smokeless tobacco is a major threat for oral cancer occurrence in the Jazan region of Saudi Arabia. This study gives a direction to conduct further longitudinal studies in the region with increased sample size representing the population in order to provide more substantial evidence.
\end{abstract}

Keywords: Oral cancer - shamma - public health - Jazan province - Saudi Arabia

Asian Pac J Cancer Prev, 16 (10), 4335-4338

\section{Introduction}

Oral cancer has been a public health concern for quite some time. Various government and nongovernment organizations are involving the community and stakeholders in order to tackle this dreadful disease. According to Warnakulasuriya, oral cancer is the eleventh most prevalent cancer in the world (Warnakulasuriya, 2009). It portrays substantial amount of variation in its spread geographically (Parkin et al., 2005). The developing nations have been reported to have more incidence of oral cancer when compared to the developed nations (Petersen, 2005; Cancela et al., 2010) of which India, South America and Oceania are mostly affected (Warnakulasuriya, 2009). The survival rate reported in oral cancer patients is the lowest when compared to the other major cancers such as the cancer of breast, skin, testis, prostate, uterus and urinary bladder (Pisani et al., 1999). The mean value reported is 0.54 for females and
0.41 for males (Berrino et al., 2003). Oral cancer ranks second after cardiovascular disease in terms of mortality; which means that the people dying from oral cancer and cardio vascular diseases are more when compared to any other diseases globally (Andisheh-Tadbir et al., 2008). The World Health Organization reports that 2 in 100,000 die due to oral cancer, in which around $90 \%-95 \%$ are diagnosed as suffering from Squamous Cell Carcinomas (SCC's) (Ame'zaga et al., 2007).

Studies conducted suggests that the risk factors which are seen to have a significant association with oral cancer are tobacco, alcohol and human papilloma virus (Lin et al., 2011a). Investigators comment that alcohol and smoking are accountable for $80 \%$ of the cases in males, $61 \%$ of the cases in females and $74 \%$ overall (Warnakulasuriya et al., 2005; Gillison, 2007 and Petersen, 2009). Tobacco in the form of a chewing material (smokeless) is also seen to be responsible for oral cancer (Lin et al., 2011b). Among the local inhabitants of Jazan region in Saudi Arabia, there is

${ }^{1}$ Faculty of Dentistry, Jazan University, Jazan, ${ }^{2}$ Department of Otolaryngology-Head and Neck Surgery, Faculty of Medicine, Jazan University, ${ }^{3}$ Oral and Maxillofacial Department, King Fahd Central Hospital, Abu Areesh, Jazan, Saudi Arabia *For correspondence: faeq_ali@yahoo.com 
a high prevalence of shamma use. Shamma is a form of smokeless tobacco in powdered form mixed with lime, ash, pepper and flavoring oils. The user quite often places it in the buccal cavity as a quid till the affect and the flavor of the substance lasts. Studies performed in Saudi Arabia have revealed frequent use of shamma among the people living in the Jazan province, which is situated in the southern tip of the Arabian Peninsula (Allard et al., 1999; Al Sanosy, 2014). Residents as young as 10-13 years of age have been reported to be indulged in the habit of shamma dipping. The appropriate percentage of the users could be much higher as the use of shamma is illegal in the country and there are chances of false reporting by the users (Salem et al., 1984; Allard et al., 1999; Halboub et al., 2012).

Currently, the literature available to demonstrate the association of shamma dipping with oral cancer is scarce. There is no study conducted in the Jazan region of Saudi Arabia, which has the highest number of oral cancer patients in the country (Allard et al., 1999). This study is designed to evaluate the association of various locally used substances especially shamma with oral cancer in Jazan region of Saudi Arabia.

\section{Materials and Methods}

\section{Ethical consideration}

An ethical approval was first obtained from the College of Dentistry, Jazan University. Later permission was obtained from the ethics committee at King Fahd Central Hospital, Jazan, Saudi Arabia to access the medical records. The patients were contacted through telephone for their agreement to participate in the study. On arrival a written and informed consent was made to sign from each participant. Written permission was also obtained from the controls for their agreement to participate in the study.

\section{Study setting and sample}

The study was based at King Fahd hospital in the Jazan province of Saudi Arabia. This is the largest hospital in the whole province and the only one to be equipped with proper diagnostic procedures for the confirmation of various oncology cases. Potentially diagnosed cases in various primary and secondary centers across the region are referred to this hospital for histological confirmation and for further follow up and treatment.

For sampling, one previous study was scrutinized involving the use of similar risk factor among the people living in Yemen, which obtained an odds ratio of 12.6 (95\%CI, 3.3-48.2) (Nasher et al., 2014). Considering this to be a minimum odds ratio that the current study should obtain to confirm the association of the use of shamma with oral cancer, a minimum sample size was calculated. The power of study was kept at $90 \%$ with $20 \%$ exposure status and $95 \%$ confidence interval. The minimum sample size calculated was 16 cases. In the current study all the available 48 cases were included so as to increase the statistical power.

The medical records for the freshly confirmed cases of the year 2014 were checked and included. Altogether 48 cases were seen to be diagnosed and histologically confirmed at the King Fahd hospital during this time period. For each observed and confirmed case of oral cancer, two controls were matched (Amtha, 2014). Controls were patients visiting the hospital following the same referral route as cases approaching various departments other than that of Oral surgery, E.N.T and Oncology. They were matched with age ( \pm 5 years $)$, gender and location.

\section{Data collection and statistical analysis}

Apart from demographic details of each participant, shamma use-its duration, khat chewing, cigarette (number of packs per day) and pipe smoking were the variables that were recorded through the means of questionnaire. The data obtained was summarized as percentages or mean \pm standard deviation (SD). Individually the potential risk factors were analyzed with chi-squared test. Multiple logistic regressions were performed to check the independent predictors of oral cancer in this cancer prone region. All the analysis was done using IBM SPSS, version 20.

\section{Results}

It is seen that the percentage of males suffering from oral cancer (18.74\%) are only slightly higher than females $(14.58 \%)$ in the total number of cases examined for the time period in which the study was conducted. Most of the people suffering from oral cancer were above 50 years of age. Surprisingly some of the cases $(1.38 \%)$ were also of a younger ( $<30$ years) age group. The table 1 which portrays the use of different risk factors among the various categorized age groups reveals that shamma use is very common among older age groups $(21.52 \%)$. It is seen that apart from khat use all the other risk factors were seen to be significantly associated with the categorized age groups. To assess the association, initially a multivariate analysis is performed (Table 2) to obtain the odds ratio without the interference of other risk factors. This revealed that shamma, cigarette and pipe smoking were significantly

Table 1. Habits of the Study Population and its Association with the Categorized Age Groups

\begin{tabular}{lcccc}
\hline Risk factors & & Age Groups & \\
& $<30$ years & $30-40$ years & $40-50$ years & P-value \\
\hline Shamma & $2(1.28 \%)$ & $8(5.55 \%)$ & $15(10.41 \%)$ & $31(21.52 \%)$ \\
Khat & 0 & $2(1.38 \%)$ & $5(3.47 \%)$ & $3(2.07 \%)$ \\
Cigarette & 0 & $5(3.47 \%)$ & $5(3.47 \%)$ & $6(4.16 \%)$ \\
Pipe smoking & 0 & $4(2.77 \%)$ & $7(4.86 \%)$ & $3(2.07 \%)$ \\
\hline
\end{tabular}

*S-Significant, NS- Not significant; a - Chi-Squared test 
Table 2. Multivariate Analysis Demonstrating the Association of Risk Factors with Oral Cancer

\begin{tabular}{|c|c|c|c|c|c|}
\hline \multirow[t]{2}{*}{ Risk factors } & \multicolumn{2}{|c|}{ Outcome } & \multirow[b]{2}{*}{ Mean \pm S.D } & \multirow[b]{2}{*}{ P-value ${ }^{a}$} & \multirow{2}{*}{$\begin{array}{c}\text { Odds Ratio } \\
(95 \% \mathrm{CI})\end{array}$} \\
\hline & Cases $(\mathrm{N}=48)$ & Controls $(\mathrm{N}=96)$ & & & \\
\hline Khat & $2(1.38 \%)$ & $8(5.55 \%)$ & $0.07 \pm 0.25$ & $0.35(\mathrm{NS})$ & $0.48(0.10-2.34)$ \\
\hline Shamma & $39(27.08 \%)$ & $17(11.80 \%)$ & $0.83 \pm 0.38$ & $0.00(\mathrm{~S})^{*}$ & $20.14(8.23-49.25)$ \\
\hline Cigarette & $10(6.94 \%)$ & $6(4.16 \%)$ & $0.11 \pm 0.31$ & $0.009(\mathrm{~S})^{*}$ & $3.95(1.34-11.63)$ \\
\hline Pipe & $9(6.24 \%)$ & $5(3.47 \%)$ & $0.10 \pm 0.30$ & $0.01(\mathrm{~S})^{*}$ & $4.20(1.32-13.34)$ \\
\hline
\end{tabular}

*S-Significant, NS- Not significant; a - Chi-Squared test

Table 3. Duration of Shamma Use with Oral cancer

\begin{tabular}{lrrr}
\hline Duration & Cases $(\mathrm{n}=48)$ & Controls $(\mathrm{n}=96)$ & P value \\
\hline$<10$ years & $6(4.16 \%)$ & $12(8.33 \%)$ & \\
$10-15$ years & $21(14.58 \%)$ & $1(0.69 \%)$ & $0.00^{*}(\mathrm{~S})$ \\
$15-20$ years & $21(14.58 \%)$ & $1(0.69 \%)$ & \\
$>$ 20 years & 0 & 0 & \\
\hline
\end{tabular}

Table 4. Multiple Logistic Regression Model Showing Predictors of Oral Cancer

\begin{tabular}{lccc}
\hline Predictors & P-value & O.R $(95 \% \mathrm{CI})$ & $\mathrm{R}^{2}$ \\
\hline Model 1: & & & 0.4 \\
Shamma & $<0.05$ & $29.30(10.33-83.13)$ & \\
Cigarette* & $<0.05$ & $6.74(2.18-20.83)$ & \\
Model 2 (Interaction): & & \\
Shamma & $<0.05$ & $37.24(12.25-113.18)$ & 0.41 \\
Cigarette* & $<0.05$ & $10.48(2.88-3.11)$ & \\
Khata Shamma & 0.02 & $0.01(0.00-0.65)$ & \\
\hline
\end{tabular}

*(number of packs per day); ${ }^{\text {- }}$ khat showing negative effect

associated with oral cancer; and that shamma scored the highest odds ratio $(\mathrm{OR}=20.14 ; 95 \% \mathrm{CI}$ 8.23-49.25). The duration of shamma use was also seen to have a significant effect on the occurrence of oral cancer (Table 3). Multiple logistic regressions with the adjustment for the covariates without interaction revealed that shamma increases the odds of having oral cancer by 29 times $(\mathrm{OR}=29.30 ; 10.33$ $83.13)$ and cigarette by 6 times $(\mathrm{OR}=6.74 ; 2.18-20.83)$ in users when compared to non-users. In the interaction model the odds ratio is increased to a significantly higher level for shamma users $(\mathrm{OR}=37.24 ; 12.25-113.18)$ and cigarette smokers $(\mathrm{OR}=10.48 ; 2.88-3.11)$. Very interestingly, the khat use is seen to have a negative effect on the disease occurrence when used along with shamma (Table 4).

\section{Discussion}

In discussing the results we observe that most of the individuals suffering from oral cancer were over 50 years of age, which is in accordance with an earlier study (Ferlay et al., 2013). Two researches conducted in Yemen which share the border with Jazan showed higher number of females suffering from oral cancer when compared to males (Sawair et al., 2007; Halboub et al., 2012). This probably justifies the almost equal percentage of male and female oral cancer cases obtained in the current study. Though cigarette smoking (number of cigarettes per day) is observed to have an effect on the occurrence of the disease, shamma (smokeless tobacco) was seen to be majorly associated with oral cancer among the study sample. Similar type of impact of shamma has also been demonstrated by Nasher (2014) and his colleagues when they performed their study in a different study setting (Nasher et al., 2014).

The smokeless tobacco recognized by the International Agency for Research on Cancer comes in different forms and also possess various range of severity (IARC, 2007). In Sweden a form of smokeless tobacco called snus is reported to have a very minimal link in causing oral cancer (Rodu and Jansson, 2004; Weitkunat et al., 2007; Lee and Hamling, 2009). But in other parts of the world such as in the African country of Sudan, it is seen that Toombak and Safa (smokeless tobacco) showed a strong association with oral cancer (Elbeshir et al., 1989; Idris et al., 1995a; 1995b). The odds ratio obtained in this study and the one that was done in Yemen (Nasher et al., 2014) is significantly higher indicating its strong carcinogenic effect. The presence of tobacco-specific nitrosamines (TSNAs) and polycyclic aromatic hydrocarbons is the key in identifying the differences in carcinogenicity of shamma, toombak, snus and various other available substances (IARC, 2012). It is obvious with the results portrayed by the current study that shamma may possesses a high amount of carcinogens when compared to the rest of the smokeless tobacco.

Jazan region is reported to have the highest number of oral cancer cases diagnosed each year in the Kingdom of Saudi Arabia (Quadri et al., 2014). A previous retrospective longitudinal study conducted from 1976 till 1995 using the data obtained at the King Faisal specialist hospital and research center in Riyadh revealed that $35.4 \%$ of all the oral cancer cases from the nation were just from one province namely, Jazan (Allard et al., 1999). Noticing the spread of this deadly disease in the region, it was necessary to initiate a study in order to gather the evidence of various potential risk factors involved within the local population. The authors report that this study is the first of its kind to assess the association of various locally used risk factors to oral cancer in the Jazan region and it is the second such study to be conducted in the Southern Arabian Peninsula (Nasher et al., 2014).

Though the study had low sample size, care was taken to make sure that the calculation supports the minimum odds ratio to define the risk factor for oral cancer. The positive aspect of the study is the recruitment of homogenous study sample to help in controlling for other risk factors of oral cancer. This is an added advantage in the investigation, as oral cancer is a complex disease with multiple factors involved in its etiology (Dos Santos Silva, 1999; Schoenbach and Rosamond, 2000).

In discussing the limitations, the authors acknowledge that the study sample is not representative of Jazan region 
and because it is a hospital based study there is always a chance of selection bias. With the aspect of minimizing the selection bias, the controls were only selected if they followed the same referral route as cases.

In conclusion, the study reveals that shamma is the major cause of oral cancer in the region. Taking the findings from the current study into consideration other studies should be designed and conducted in future with the involvement of a larger sample size. The public health practitioners in the region should be actively involved in conducting and implementing various prevention strategies in this cancer prone region.

\section{References}

Allard WF, De Vol EB, Te OB (1999). Smokeless tobacco (shamma) and oral cancer in Saudi Arabia. Community Dent Oral Epidemiol, 27, 398-5.

Al-Sanosy RM (2014). Smokeless tobacco (shammah) in Saudi Arabia: a review of its pattern of use, prevalence, and potential role in oral cancer, Asian Pac J Cancer Prev, 15, 6477-3.

Ame'Zaga J, Herrero L, Zubizarreta J, et al (2007). Diagnostic efficacy of sentinel node biopsy in oral squamous cell carcinoma: Cohort study and meta-analysis. Med Oral Patol Oral Cir Bucal, 12, 235-3.

Amtha R, Razzak IA, Basuki B, et al (2014). Tobacco (kretek) smoking, betel quid chewing and risk of oral cancer in a selected jakarta population. Asian Pac J Cancer Prev, 15, 8673-8.

Andisheh-Tadbir A, Mehrabani D, Taghi H (2008). Epidemiology of squamous cell carcinoma of the oral cavity in Iran. $J$ Craniofacial Sur, 19, 1699-2.

Berrino F, De Angelis R, Sant M, et al (2003). The Eurocare Study: strenghts, limitations and perspectives of populationbased, comparative survival studies. Ann Oncol, 14, 9-3.

Cancela MDC, Voti L, Guerra-Yi MEA (2010). Oral cavity cancer in developed and in developing countries: Populationbased incidence. J Sci Specialities Head Neck, 32, 357-7.

Dos Santos Silva I (1999). Cancer Epidemiology: Principles and Methods. Lyon: International Agency for Research on Cancer, Website: http://www.iarc.fr/en/publications/pdfs online/epi/cancerepi/CancerEpi.pdf.

El Beshir EI, Abeen HA, Idris AM, et al (1989). Snuff dipping and oral cancer in Sudan: a retrospective study. Br J Oral Maxillofac Surg, 27, 243-8.

Ferlay J, Soerjomataram I, Ervik M, et al (2013). Cancer Incidence and Mortality Worldwide: IARC CancerBase No.11. GLOBOCAN 2012, 1.

Gillison M (2007). Current topics in the epidemiology of oral cavity and oropharyngeal cancers. Head Neck, 29, 779-2.

Halboub ES, Abdulhuq M, Al-Mandili A (2012). Oral and pharyngeal cancers in Yemen: a retrospective study. East Mediterr Health J, 18, 985-1.

IARC (2007). Working Group on the Evaluation of Carcinogenic Risks to Humans. International Agency for Research on Cancer. Smokeless tobacco and some tobacco-specific N-nitrosamines. World Health Organization; distributed by WHO Press, Lyon, France.

IARC (2012). Working Group on the Evaluation of Carcinogenic Risks to Humans, Personal habits and indoor combustions: A review of human carcinogens. International Agency for Research on Cancer. Smokeless tobacco and some tobaccospecific N-nitrosamines, World Health Organization; distributed by WHO Press, Lyon, France, 100.

Idris AM, Ahmed HM, Malik MO (1995a). Toombak dipping and cancer of the oral cavity in the Sudan: a case-control study. Int J Cancer, 63, 477-80.

Idris AM, Ahmed HM, Mukhtar BI, et al (1995b). Descriptive epidemiology of oral neoplasms in Sudan 1970-1985 and the role of toombak, Int J Cancer, 61, 155-8.

Lee PN, Hamling J (2009). Systematic review of the relation between smokeless tobacco and cancer in Europe and North America, BMC Med, 7, 36-1.

Lin W, Jiang RS, Wu SH, et al (2011a). Smoking, alcohol, and betel quid and oral cancer: a prospective cohort study. $J$ Oncol, 11, 525-6.

Lin WJ, Jiang RS, Wu SH, et al (2011b). Smoking, alcohol, and betel quid and oral cancer: a prospective cohort study. J Oncol, 11, 1-5.

Nasher AT, Al-Hebshi NN, Al-Moayad EE, et al (2014). Viral infection and oral habits as risk factors for oral squamous cell carcinoma in Yemen: a case-control study. Oral Surgery Oral Medicine Oral Pathology Oral Radiology, 118, 566-2.

Parkin D, Bray F, Ferlay J, et al (2005). Global cancer statistics 2002. CA Cancer J Clin, 55, 74-8.

Petersen PE (2005). Strengthening the prevention of oral cancer: the WHO perspective. Community Dentistry Oral Epidemiol, 33, 397-9.

Petersen PE (2009). Oral cancer prevention and control-The approach of the World Health Organization. J Oral Oncol, 45, 454-40.

Pisani P, Parkin D, Bray F, et al (1999). Estimates of the worldwide mortality from 25 cancers in 1990. Int J Cancer, 83, 870-3.

Quadri MFA, Saleh SM, AlSanosy, et al (2014). Effectiveness of an Intervention Program on Knowledge of Oral Cancer among the Youth of Jazan Saudi Arabia. Asian Pac J Cancer Prev, 15, 1913-8.

Rodu B, Jansson C (2004). Smokeless tobacco and oral cancer: a review of the risks and determinants. Crit Rev Oral Biol Med, 15, 252-3.

Salem G, Juhl R, Schiodt T (1984). Oral malignant and premalignant changes in 'Shammah'-users from the Gizan region Saudi Arabia. Acta Odontol Scand, 42, 41-5.

Sawair FA, Al Mutwakel A, Al Eryani K, et al (2007). High relative frequency of oral squamous cell carcinoma in Yemen: qat and tobacco chewing as its aetiological background, Int J Environ Health Res, 17, 185-5.

Schoenbach V, Rosamond VD (2000). Multicausality. Effect modification. Understanding the Fundamental of Epidemiology-An Evolving Text. Chapel Hill: University of North Carolina, 8, 381-2.

Warnakulasuriya S (2009). Global epidemiology of oral and oropharyngeal cancer, Oral Oncol, 45.

Warnakulasuriya S, Sutherland G, Scully C (2005). Tobacco, oral cancer and treatment of dependence, Oral Oncol, 41, 244-60.

Weitkunat R, Sanders E, Lee PN (2007). Meta-analysis of the relation between European and American smokeless tobacco and oral cancer, BMC Public Health, 7, 334-1. 